\title{
Mechanical and Electrical Failure of Silver Nanowire Electrodes: A Scale Bridging In Situ Electron Microscopy Study
}

\author{
N. Schrenker ${ }^{1 *}$, P. Schweizer ${ }^{1}$, M. Moninger ${ }^{1}$, N. Karpstein ${ }^{1}$, M. Mačković ${ }^{1}$, G. D. Spyropoulos ${ }^{2}$, M. $^{2}$ \\ Göbelt $^{3}$, S. Christiansen ${ }^{3}$, C. J. Brabec ${ }^{2}$, and E. Spiecker ${ }^{1 *}$ \\ ${ }^{1 .}$ Center for Nanoanalysis and Electron Microscopy (CENEM) and Institute of Micro- and \\ Nanostructure Research (WW9), Friedrich-Alexander University Erlangen-Nuremberg (FAU), \\ Germany. \\ 2. ZAE Bayern: Bavarian Center for Applied Energy Research, Erlangen, Germany. \\ ${ }^{3 .}$ Max Planck Institute for the Science of Light, Erlangen, Germany. \\ * Corresponding author: Nadine.Schrenker@fau.de, Erdmann.Spiecker@fau.de
}

In modern electronics there is an increasing demand for reliable transparent electrodes (TE), which can withstand mechanical loading without sacrificing electrical performance. Application examples for TE are organic solar cells (OSCs), touch panels, organic light emitting diodes (OLEDs) or smart clothing. Indium tin oxide (ITO) is the most commonly used material for TEs. However, drawbacks of ITO are the relatively low abundance of indium and costly fabrication procedures based on vacuum deposition techniques. Silver nanowire (Ag NW) networks are a highly promising alternative, since they not only combine low sheet resistance with high optical transmittance but, moreover, can be fabricated by lowcost solution-based methods. Most importantly, regarding applications in flexible electronics, Ag NW networks show a much better mechanical flexibility than ITO, as revealed, e.g., in macroscopic bending tests [1]. The interplay between microscopic failure mechanisms and functional properties of Ag NW electrodes are elucidated in this work by a scale-bridging in situ approach starting from mechanical testing of single NWs up to combined mechanical and electrical testing of $\mathrm{NW}$ electrodes in transmission and scanning electron microscopy, respectively.

The Ag NWs used in the present work possess the characteristic five-fold twin structure with diameters ranging from $30 \mathrm{~nm}$ to $90 \mathrm{~nm}$. Ag NW networks are fabricated via doctor blading on flexible PET foils. PET was chosen, as it is the common substrate for flexible OSCs [2]. For in situ small scale testing of single nanowires and nanowire networks in tensile and bending mode samples are fabricated using a Focused Ion Beam (FIB).

On the nanometer scale in situ scanning TEM tensile tests of single Ag NWs reveal nucleation and propagation of mainly partial dislocations on different $\{111\}$ slip planes. Upon further straining, the wires show localized deformation and necking before fracture occurs. The yield strength reveals a clear size effect and is ten times higher compared to bulk silver. Going up in scale, in operando SEM tensile tests in conjunction with resistance measurements were carried out on Ag NW networks on PET to correlate their electrical and mechanical performance (Figure 1). The results show that the wire orientation w.r.t. the straining direction is highly critical and determines the deformation mode and corresponding failure mechanism. Wires in straining direction fail in tensile mode by necking, like in the single wire test experiment. In contrast, wires oriented perpendicular to the straining direction deform by bending as well as kinking and maintain the topological properties of the network up to higher strains. The kinks develop sharp angles of almost $90^{\circ}$. HRTEM analysis of kinks revealed the nucleation of full dislocations, small-angle grain boundaries, stacking faults and chevron defects (Figure 2). At $20 \%$ strain the increase in relative resistance is about nine times higher if the wires are oriented parallel to the 
straining direction compared to the perpendicular orientation. Consequently, the network anisotropy due to the applied coating parameters can be utilized to sustain the high conductivity level during straining and increase the lifetime of flexible devices [3].

\section{References:}

[1] S. De et al., ACS Nano 3 (2009), p. 1767-1774.

[2] L. Lucera et al., Energy Environ. Sci. 9 (2016), p. 89-94.

[3] Financial support from the German Research Foundation (DFG) via the Research Training Group GRK 1896 "In situ microscopy with electrons, X-rays and scanning probes” is gratefully acknowledged.
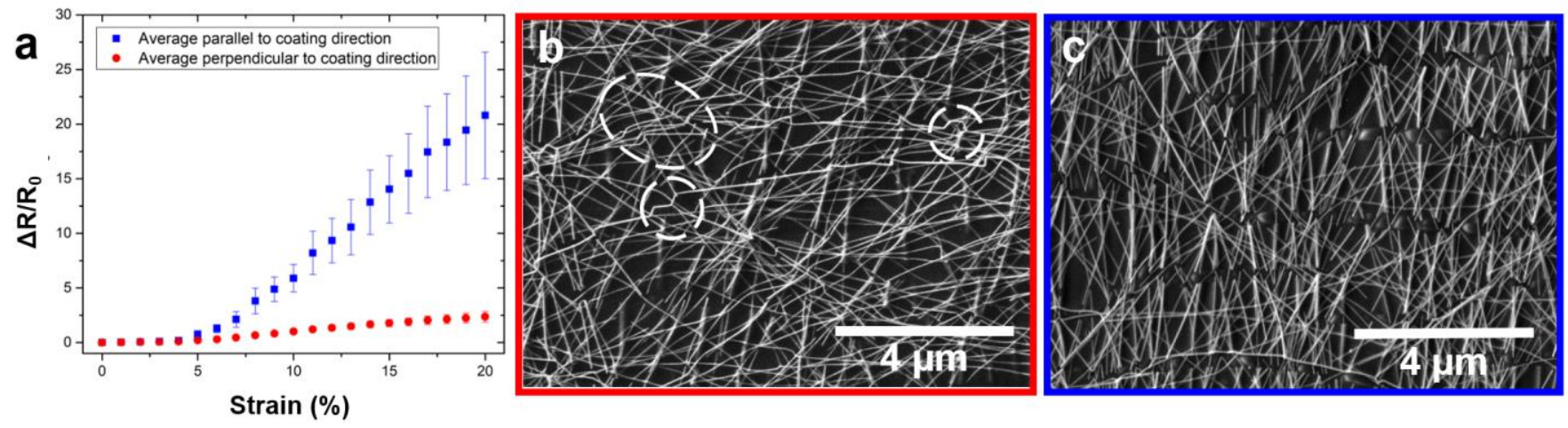

Figure 1. (a) Relative resistance change versus strain for two batches of networks (d=35 $\mathrm{nm})$, where the coating direction is parallel (blue) respectively perpendicular (red) to the straining direction. (b) SEM image of a network after tensile deformation up to $20 \%$ strain with straining direction (vertical) perpendicular to the coating direction (horizontal). In (c) straining and coating direction of the network are parallel (both vertical).
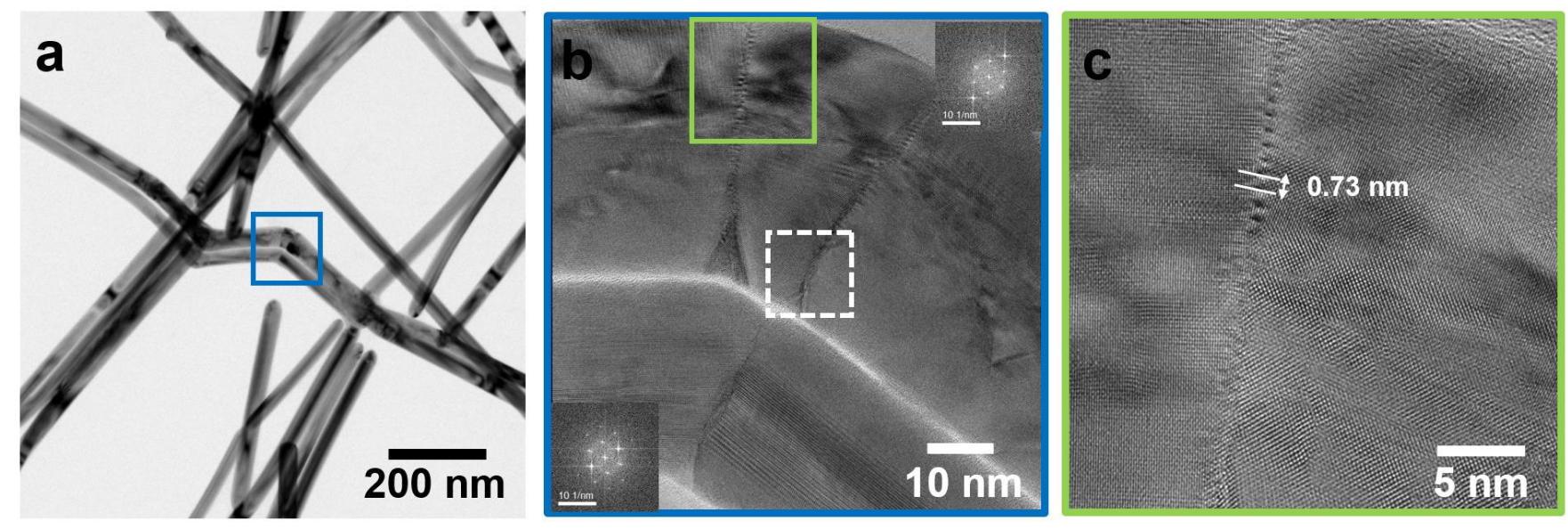

Figure 2. (a) TEM BF image of a Ag NW network strained perpendicular to the coating direction (25\% strain) revealing characteristic kink formation. (b) Close-up of the kinked wires in (a). The inserts show the FFT of the top wire on the left and right side of the boundary in the area marked with a white dashed square. (c) HRTEM image of the wire in (b) marked with a green square depicting single dislocations at the grain boundary. 